\title{
Response from CLOTHES Trial team
}

Dear Editor,

We would like to respond to the recent letter by Wüthrich and colleagues.[1]

As Wüthrich et al. have used silk garments within their hospital clinic for many decades it is unsurprising they so strongly believe in the value of these products. Unfortunately, the extent of their investment has seemingly left them unable to countenance information that does not accord with their own experience. Indeed, their correspondence aptly illustrates the need to scientifically evaluate the efficacy of treatments in adequately-powered randomised controlled trials, conducted from a position of equipoise, and therefore minimise the potential for conflicts and bias.

Wüthrich and colleagues, seemingly unfamiliar with commonly operated Open Access models used by journals, make reference to PLOS Medicines publishing fees. For their benefit, let us firstly state that PLOS Medicine, a distinguished journal, accepts just 3\% of submitted papers. Secondly, following acceptance article authors can pay fees to the journal to allow the content to be accessed by the public for free. This is not the same as paying for a paper to be accepted.

The information that Wüthrich and colleagues claim to be missing from the PLOS Medicine paper, is in fact all available. The authors state that the results of CLOTHES was contrary to previous studies. Prior to CLOTHES, the evidence on silk clothing for eczema was considered low quality and insufficient to guide practice when evaluated in three systematic reviews.[2-4] The limited evidence prompted the UK National Institute for Health Research to commission the CLOTHES study to inform practice.[5] The authors then state that our eligibility criteria meant that some with mild eczema were included. Our eligibility criteria were pre-defined in the trial protocol which was publicly available before recruitment started. They were never challenged during peer-review of the protocol or after publication.[6] Our choice of eligibility criteria did not bias the results or change the study conclusions. Sensitivity analysis that explored the impact of different eczema severities at baseline found no evidence to suggest that those with more severe disease were more likely to benefit from silk garments. The baseline severity information requested by Wüthrich et $a l$ is available in Table 2 , and implications of enrolling more children with mild disease than expected were explained in the discussion.

The authors suggest that adherence during the trial does not reflect how silk clothes are used in practice. On the contrary, our trial attempted to reflect how the garments would be used in real life. Children were asked to wear the garments as often as possible (day and night) for a period of 6 months. Children wore the garments for a median of $81 \%$ of nights and $34 \%$ of days. A separate analysis showed that there was no evidence of benefit from wearing the clothing more often.

Our interpretation of the secondary outcome of eczema symptoms is also questioned. It was clear from talking to eczema patients before the trial that expectation of benefit from silk clothing was high, thus increasing the likelihood of information bias for patient-reported outcomes given the intervention allocation was not blinded. The CLOTHES trial was therefore designed as an investigator-blind trial, whereby the primary outcome (severity using EASI) was assessed by trained and blinded research nurses. The initial small benefit shown on the POEM symptom scale was not sustained. Patient-reported results were fully discussed in our paper and considered when presenting the trial conclusions. 
We believe that the CLOTHES trial provides the first high-quality, independent evidence on the use of silk clothing for eczema. We hope that these results assist healthcare providers and people with eczema in making informed choices.

Professor Kim S Thomas on behalf of the CLOTHES Trial Team

Professor of Applied Dermatology Research

Centre of Evidence Based Dermatology

University of Nottingham

This project was funded by the National Institute for Health Research (NIHR) Health Technology Assessment programme (project number 11/65/01). The views and opinions expressed herein are those of the authors and do not necessarily reflect those of the Health Technology Assessment programme, NIHR, NHS or the Department of Health.

\section{References}

[1] Wuthrich B, Senti G, Kundig TM. Letter to the Editor: Consensus-based European guidelines for treatment of atopic eczema (atopic dermatitis) in adults and children: part II. J Eur Acad Dermatol Venereol. 2018.

[2] Vlachou C, Thomas KS, Williams HC. A case report and critical appraisal of the literature on the use of DermaSilk in children with atopic dermatitis. Clin Exp Dermatol. 2009;34; e901-903.

[3] Bath-Hextall FJ, Birnie AJ, Ravenscroft JC, Williams HC. Interventions to reduce Staphylococcus aureus in the management of atopic eczema: an updated Cochrane review. $\mathrm{Br} \mathrm{J}$ Dermatol. 2010;163; 12-26.

[4] Lopes C, Silva D, Delgado L, Correia O, Moreira A. Functional textiles for atopic dermatitis: a systematic review and meta-analysis. Pediatr Allergy Immunol,. 2013;24; 603-613.

[5] Thomas KS, Bradshaw LE, Sach TH, Cowdell F, Batchelor JM, Lawton S, et al. Randomised controlled trial of silk therapeutic garments for the management of atopic eczema in children: the CLOTHES trial. Health Technol Assess. 2017;21; 1-260.

[6] Harrison EF, Haines RH, Cowdell F, Sach TH, Dean T, Pollock I, et al. A multi-centre, parallel group superiority trial of silk therapeutic clothing compared to standard care for the management of eczema in children (CLOTHES Trial): study protocol for a randomised controlled trial. Trials. 2015;16; 390. 